\title{
IMPLEMENTATION OF AUTOMATIC CRACK EVALUATION USING CRACK FUNDAMENTAL ELEMENT
}

\author{
Yichang (James) Tsai, Chenglong Jiang, and Zhaohua Wang \\ Georgia Institute of Technology \\ 790 Atlantic DR. NW, Atlanta, GA 30332, USA
}

\begin{abstract}
Crack evaluation is a vital component in the pavement condition survey. However, researchers have had difficulty automatically mimicking the in-field evaluation practice to achieve a fully automated pavement crack evaluation due to the diversity of real-world crack patterns and the complexity of crack definitions in an agency's survey protocol. The multi-scale crack analysis model based on a Crack Fundamental Element is developed to topologically represent complex crack patterns and provide systematic crack properties to support crack classification. Utilizing this model, this study implements an automatic crack classification and quantification method for use on the state highways in Georgia, United States. Both image-based validation and field validation are conducted with the pavement experts from the Georgia Department of Transportation (GDOT) on State Route 236 in Atlanta, and the proposed method achieves a classification accuracy of $92.2 \%$ for load cracking and $98.1 \%$ for $\mathrm{B} / \mathrm{T}$ cracking.
\end{abstract}

Index
Fundamental Element $\quad$ Crack Classification, Crack

\section{INTRODUCTION}

Cracking is one of the most common types of pavement distresses. It is usually caused by constant overloading, asphalt aging, environmental impact, and improper structural design, etc. The proper treatment of pavement cracks is important for cost-effective pavement maintenance. Many transportation agencies in the US, including the Georgia Department of Transportation (GDOT), the Texas Department of Transportation (TxDOT), etc., have invested major resources into their pavement cracking surveys to enhance their maintenance decision-making. In recent years, automated crack data collection and evaluation has gained attention among transportation agencies and researchers.

Significant research efforts have been devoted to the development of high-resolution sensing techniques and accurate crack detection algorithms. However, the raw data and crack detection results cannot be used directly by transportation agencies. Crack classification and quantification is critical for linking crack detection results to pavement maintenance decision-making, yet studies on crack classification are limited. Most existing literature investigates similar physical crack characteristics for classification purposes. Crack orientation is the most common characteristic. Past studies utilize different approaches for capturing crack orientations, e.g. histogram analysis [1-4] and wavelet and radon transform [5], etc. and use them to differentiate longitudinal cracking, transverse cracking, and diagonal cracking, etc. Crack amount is another common characteristic, which is usually represented by the number of crack pixels/tiles; it is used to differentiate the crack types with high density, such as alligator cracking $[2,6]$.

However, the real-world crack protocols used by transportation agencies have much more complicated crack definitions. For example, Figure 1 illustrates load cracking and block/transverse $(\mathrm{B} / \mathrm{T})$ cracking at different severity levels in GDOT's pavement cracking survey. The classification of these crack types and severity levels involves human identification of complex and diverse crack patterns, and it is insufficient to simply rely on crack orientation and amount. In order to represent real-world, complex crack patterns, Tsai et al. [7] proposed the multiscale Crack Fundamental Element (CFE) model to provide rich and systematic crack properties for crack classification. Based on this previously proposed model, this paper develops an automatic crack evaluation method for load and $\mathrm{B} / \mathrm{T}$ cracking, which are the two most common types of pavement surface distresses in Georgia, USA, and presents its implementation on state highways.

\section{MULTI-SCALE CRACK ANALYSIS BASED ON CRACK FUNDAMENTAL ELEMENTS}

Crack pattern is crucial for differentiating crack types and severity levels in transportation agencies' pavement survey practices. Tsai, et al. [7] defines a Crack Fundamental Element (CFE) to present these topological patterns by clustering a group of cracks based on their location, orientation, length, etc. It starts from groups of pixels that form approximate linear structures as the initial CFEs, then gradually clusters into higher scales. Figure 2 briefly shows the logic of multi-scale crack properties extraction. 


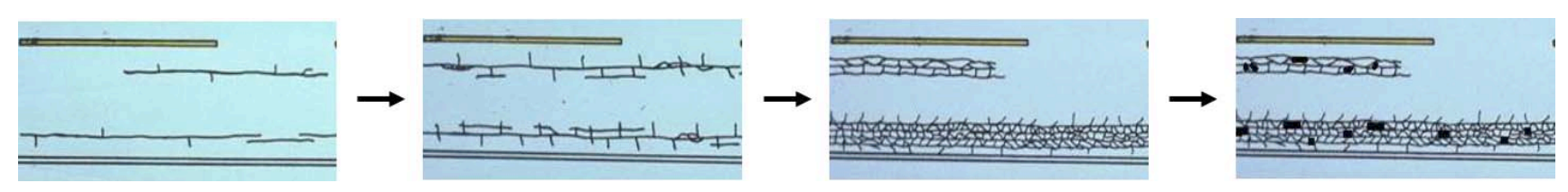

(a) Load cracking from severity level 1 to 4 (from left to right)
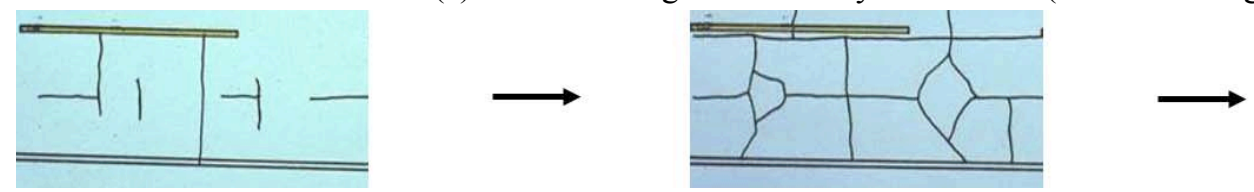

(b) $\mathrm{B} / \mathrm{T}$ cracking from severity level 1 to 3 (from left to right)

Figure 1. Illustration of load cracking and $\mathrm{B} / \mathrm{T}$ cracking at different severity levels

This multi-scale CFE model provides rich crack properties at three different scales to support the development of an automatic crack classification method:

1) Fundamental crack properties focus on each crack segment and describe the fundamental and physical properties of cracks, such as crack location, length, width, orientation, etc.

2) Aggregated crack properties focus more on crack patterns inside the clustered CFE and represent how cracks interact with each other, including intersections and polygons.

3) CFE cluster geometrical properties treat each CFE as a whole and describe its overall properties. These geometrical properties are also used to cluster CFEs from low scale to high scale.

$\circ$ CFE length

Length (L) is defined as the length of the major axis of the ellipse that has the same normalized second central moments as the group of crack curves that are clustered together in a CFE:

$$
L=2 \times \sqrt{2\left(\left(\mu_{x x}+\mu_{y y}\right)+\Delta\right)}
$$

where $\mu_{x x}=\frac{\sum_{i=1}^{N} x_{i}^{2}}{N}, \mu_{y y}=\frac{\sum_{i=1}^{N} y_{i}^{2}}{N}, \mu_{x y}=\frac{\sum_{i=1}^{N} x_{i} y_{i}}{N}$, and $\Delta=\sqrt{\left(\mu_{x x}-\mu_{y y}\right)^{2}+4 \times \mu_{x y}^{2}}$ are the second moments of the crack curves in that CFE.

- CFE Width

Width (W), similarly, is defined as the length of the minor axis of the ellipse that has the same normalized second central moments as the group of crack curves that are clustered together in a CFE:

$$
W=2 \times \sqrt{2\left(\left(\mu_{x x}+\mu_{y y}\right)-\Delta\right)}
$$

- CFE Center

Center (C) is defined as the centroid of crack curves in the CFE:

$$
C=\left(\mu_{x}, \mu_{y}\right)=\left(\frac{\sum_{i=1}^{N} x_{i}}{N}, \frac{\sum_{i=1}^{N} y_{i}}{N}\right)
$$

- CFE Orientation

Orientation $(\mathrm{O})$ is a measure of the CFE direction relative to the horizontal axis of the data sample:

$$
O= \begin{cases}\frac{180}{\pi} \times \tan ^{-1}\left(\frac{\mu_{x x}+\mu_{y y}+\Delta}{2 \mu_{x y}}\right), & \text { if } \mu_{x x}<\mu_{y y} \\ \frac{180}{\pi} \times \tan ^{-1}\left(\frac{2 \mu_{x y}}{\mu_{x x}+\mu_{y y}+\Delta}\right), & \text { if } \mu_{x x} \geq \mu_{y y}\end{cases}
$$

As shown in Figure 2, from the bottom, the model represents more of the physical characteristics of pavement cracks; from the top, it tends to mimic the pavement engineers' manual evaluation procedure in the field (from macro to micro level observation). When experienced pavement engineers conduct a condition survey, they do not usually measure the crack width and depth first; instead, they first identify a group of cracks that should be clustered together; then, they look at the crack pattern inside the cluster, and, finally, they measure the physical and fundamental crack properties if necessary. By clearly defining three scales of crack representation, this approach can better incorporate both physical crack characteristics and the logic of human judgment and systematically provide useful crack properties.

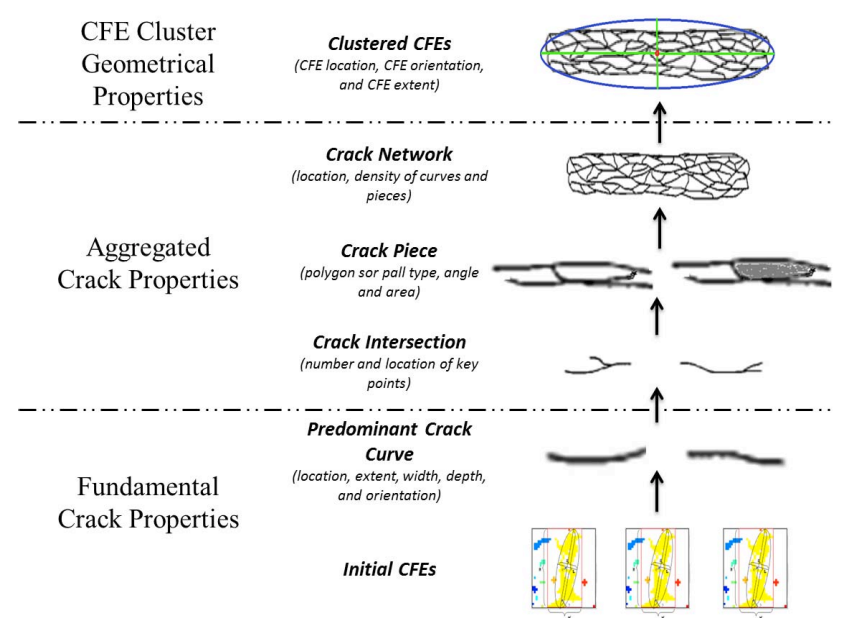

Figure 2 Multi-scale crack properties from CFE model 


\section{AUTOMATIC ASPHALT CRACK EVALUATION USING CFE MODEL}

Utilizing the crack properties from the CFE model, this section presents the method for automatically classifying and measuring load cracking and $\mathrm{B} / \mathrm{T}$ cracking, including their severity levels. The flowchart of the proposed method is shown in Figure 3. It takes the automated detected crack map as the input; the crack detection algorithm is not within the scope of this paper.

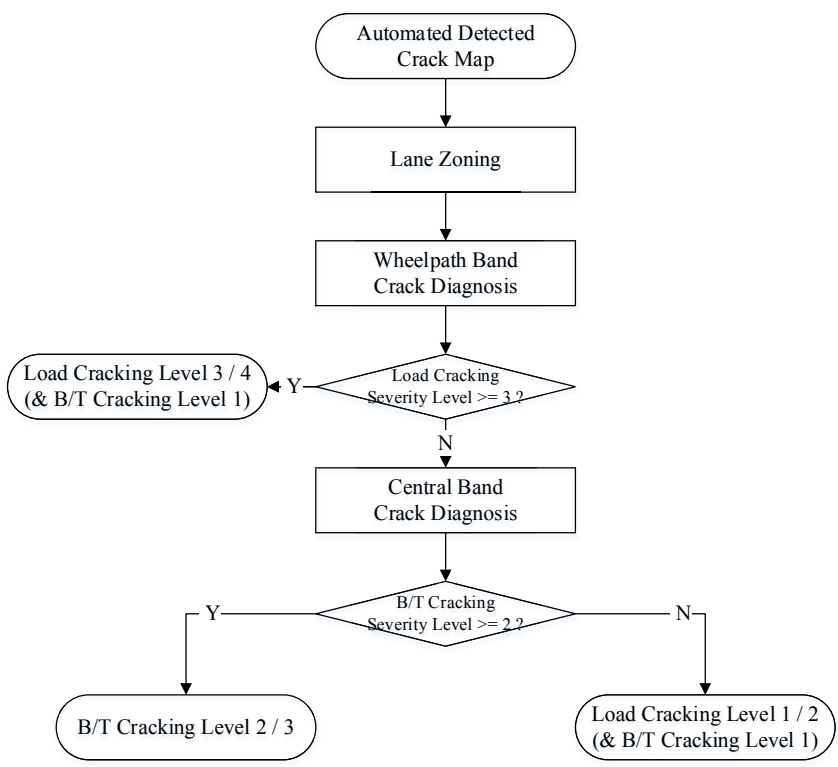

Figure 3. Flowchart of the proposed method

The first step in this method is lane zoning, which divides the pavement surface into five different bands with reference to the locations of lane marking, including one central band, two wheelpath bands, and two edge bands. The wheelpath bands and central band are the regions of interest in this study.

After the lane zoning step, crack diagnosis is conducted on the wheelpath bands and the central band. If the clustered CFE falls into any of these bands, it may indicate the presence of either load cracking or B/T cracking. A list of crack properties is calculated following the CFE model to represent the topological pattern of the crack map. Table 1 shows the crack properties used in this study.

These crack properties are the input features to further determine the crack type and severity levels through a supervised learning approach. The data whitening is applied to counterbalance the potential feature scaling issue, and the nonlinear multiclass support vector machine (SVM) with Gaussian kernel is selected as the classifier. Besides SVM, a few classifiers have also been explored and compared through the study, including logistic regression and random forest; however, preliminary results show that the impacts of different classifiers are less significant compared to different feature selections.

Table 1. Crack properties from wheelpath and central bands

\begin{tabular}{|c|c|}
\hline \multirow{7}{*}{$\begin{array}{c}\text { Fundamental Crack } \\
\text { Properties }\end{array}$} & Length of longitudinal cracks \\
\hline & Length of total cracks \\
\hline & Ratio of longitudinal to total length \\
\hline & Number of initial CFEs \\
\hline & Number of continuous crack lines \\
\hline & Average crack width \\
\hline & Maximum crack width \\
\hline \multirow{3}{*}{$\begin{array}{l}\text { Aggregated Crack } \\
\text { Properties }\end{array}$} & Number of crack intersect points \\
\hline & Area of surface loss \\
\hline & $\begin{array}{c}\text { Crack distribution based on } \\
\text { orientations }\end{array}$ \\
\hline \multirow{2}{*}{$\begin{array}{c}\text { CFE Cluster } \\
\text { Geometrical Properties }\end{array}$} & Length of clustered CFEs \\
\hline & Width of clustered CFEs \\
\hline
\end{tabular}

\section{EXPERIMENTAL RESULTS}

The proposed method is implemented on the state highways in Georgia. A field validation is conducted on State Route 236 in Atlanta, Georgia, which has an excessive amount of load cracking and B/T cracking. In this study, the Laser Crack Measurement System [8] is used to collect the pavement data, and crack detection is conducted using the analyzer software along with the system.

An image-based validation is first conducted. With the help of GDOT pavement experts, each pavement image is visually reviewed, and the presence and severity level of load cracking and B/T cracking are manually labeled. The data from each crack type and severity level are randomly separated into two sets: $70 \%$ of the data are used for training purposes, while $30 \%$ are used for testing. The experimental results are presented as follows.

For load cracking, the test set contains 701 images, as shown in Table 2. The method performs quite well and has an overall high classification accuracy of about $92.2 \%$. From the perspective of recall, a larger portion of Severity Level 2 is not correctly classified compared to other severity levels. Load cracking in Severity Level 2 is a transition stage in the crack pattern development, which features short transverse cracks and their intersection with the original longitudinal crack from Severity Level 1. Many of these cracks are thin and shallow, and it is difficult, for crack detection algorithms and even human surveyors, to capture the crack pattern completely. Therefore, the feature calculation for these crack patterns is sometimes biased, and further leads to a false classification. From the perspective of precision, the classification for Severity Level 2 is quite low, which is mainly due to the clear difference between the sample sizes. 
The imbalanced training data may also have an impact on the performance: the classification accuracy on the majority classes tends to be higher, while the accuracy on the minority classes (Severity Level 2 and higher) becomes lower. More data on the minority classes are suggested to further optimize the classification boundary and improve the classifier robustness.

Table 2. Accuracy on load cracking classification

\begin{tabular}{|c|c|c|c|c|c|c|c|}
\cline { 3 - 8 } \multicolumn{2}{c|}{} & \multicolumn{5}{c|}{ Predicted Level } & \multicolumn{2}{c|}{} \\
\cline { 2 - 8 } \multicolumn{2}{c|}{} & None & $\begin{array}{c}\text { Level } \\
1\end{array}$ & $\begin{array}{c}\text { Level } \\
2\end{array}$ & $\begin{array}{c}\text { Level } \\
3 \& 4\end{array}$ & Total & $\begin{array}{c}\text { Recall } \\
(\%)\end{array}$ \\
\hline \multirow{4}{*}{$\begin{array}{c}\text { Actual } \\
\text { Level }\end{array}$} & None & 247 & 15 & 0 & 0 & 262 & 94.3 \\
\cline { 2 - 8 } & Level 1 & 10 & 317 & 20 & 0 & 347 & 91.4 \\
\cline { 2 - 8 } & Level 2 & 0 & 6 & 42 & 2 & 50 & 84.0 \\
\cline { 2 - 8 } & Level 3\&4 & 0 & 0 & 2 & 40 & 42 & 95.2 \\
\hline \multirow{4}{*yyyyyyy}{} & Total & 257 & 338 & 64 & 42 & 701 & \\
\cline { 2 - 8 } & $\begin{array}{c}\text { Precision } \\
(\%)\end{array}$ & 96.1 & 93.8 & 65.6 & 95.2 & & 92.2 \\
\hline
\end{tabular}

For $\mathrm{B} / \mathrm{T}$ cracking, the method achieves an overall accuracy of $98.1 \%$ on the test set of 368 images. Figure 4 shows several example results for the automatic classification and quantification for both load cracking and $\mathrm{B} / \mathrm{T}$ cracking.
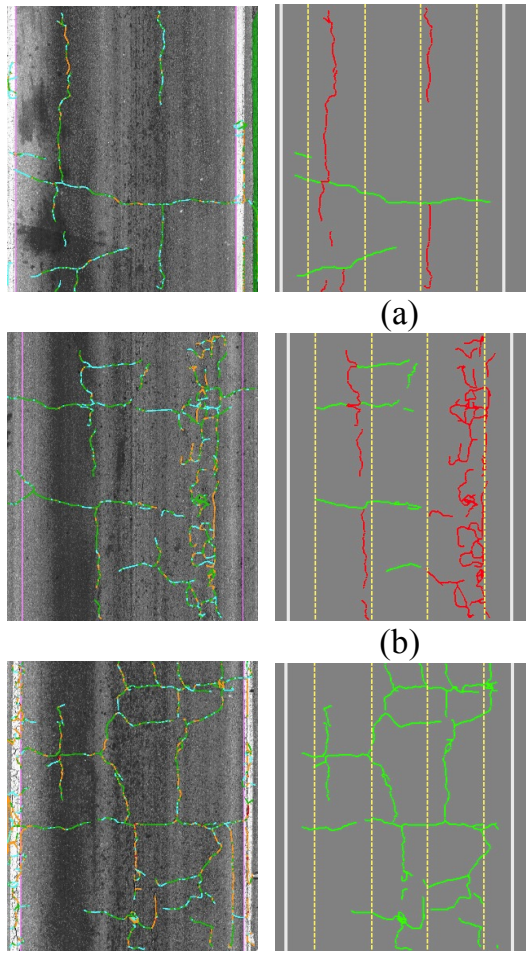

(c)

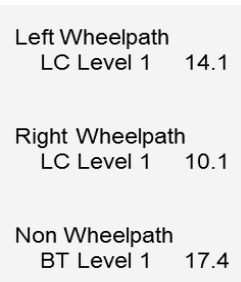

(a)
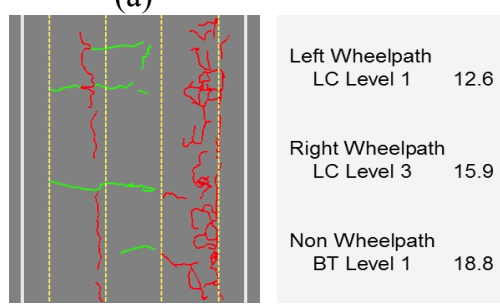

BT Level 19

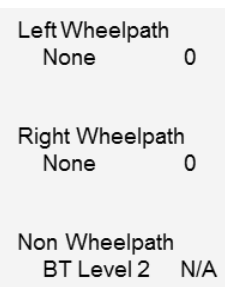

BT Level $2 \quad \mathrm{~N} / \mathrm{A}$
Figure 4. Example results of automatic crack evaluation, from left to right: detected crack map, load cracking (red) and B/T cracking (green), and final outcomes (unit: foot)
Besides the image-based validation, field validation is conducted to further validate the accuracy of the automatic crack evaluation. Four $100-\mathrm{ft}$. pavement sections are selected from the road. GDOT pavement experts visually identify the crack types and severity levels in the field, and the crack extents are precisely measured using a measuring wheel. The deduct values (corresponding to the crack type, severity level, and extent) are calculated and compared between the manual survey and the automatic crack evaluation. Based on the experimental results for the four selected sites, the average absolute difference on overall deducts between automatic crack evaluation and field measurement is 3.25 , which is within the error tolerance $( \pm 5$ deduct points) in GDOT's current survey practice. The difference is primarily caused by the failure in the crack detection stage, including

1) Missing fine cracks (usually around $1 \mathrm{~mm}$ wide, as shown in Figure 5 (a)), and

2) Incomplete crack pattern (as shown in Figure 5 (b)).

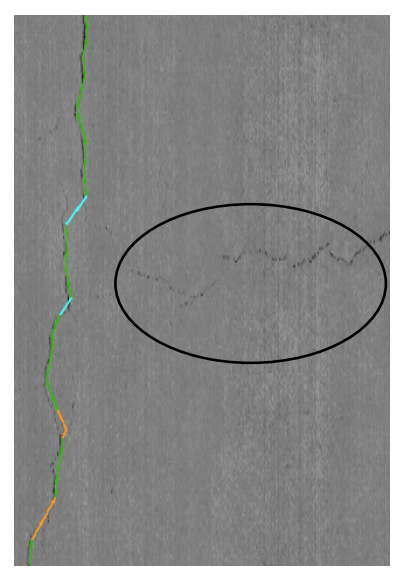

(a)

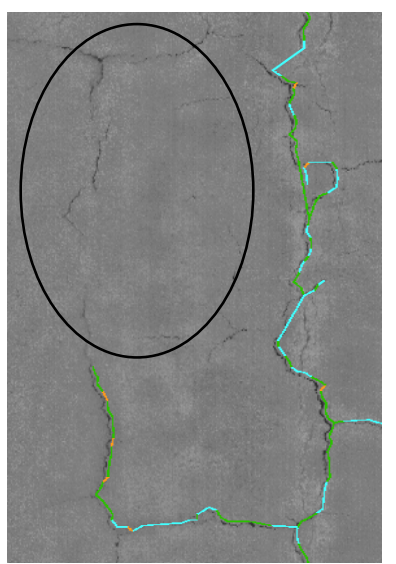

(b)
Figure 5. Differences between automatic evaluation and manual survey are mainly caused by (a) missing fine cracks and (b) incomplete topological pattern

\section{CONCLUSIONS AND FUTURE WORK}

In this paper we proposed an automatic crack evaluation method using the multi-scale CFE model. The proposed method is independent of data acquisition techniques and is adaptable to different crack detection algorithms with minor modification. Currently, we are applying the proposed method on state highways in Georgia for crack classification and quantification following GDOT's pavement survey practice. It has demonstrated a strong capability in experimental tests and is promising for transforming sensing data and crack detection results into useful decision support information. For future work, the multi-scale CFE model and the proposed method can be extended to different pavement survey protocols. Improvements in the crack detection algorithms can also contribute to a more accurate automatic crack evaluation. 


\section{REFERENCES}

[1] H. D. Cheng, J.-R. Chen, C. Glazier, and Y. G. Hu, "Novel approach to pavement cracking detection based on fuzzy set theory," Journal of Computing in Civil Engineering, vol. 13, pp. 270-280, 1999.

[2] B. J. Lee and H. D. Lee, "Position-invariant neural network for digital pavement crack analysis," Computer-Aided Civil and Infrastructure Engineering, vol. 19, pp. 105-18, 2004.

[3] E. Salari and G. Bao, "Pavement distress detection and severity analysis," in Proceedings of SPIE Image Processing: Machine Vision Applications IV, San Francisco Airport, California, USA, 2011.

[4] T. Saar and O. Talvik, "Automatic asphalt pavement crack detection and classification using neural networks," in Proceedings of the 12th Biennial Baltic Electronics Conference, 2010, pp. 345-348.
[5] J. Zhou, P. Huang, and F.-P. Chiang, "Waveletbased pavement distress classification," Transportation Research Record, pp. 89-98, 2005.

[6] W. Xiao, X. Yan, and X. Zhang, "Pavement Distress Image Automatic Classification Based on DENSITY-Based Neural Network," in Proceedings of Rough Sets and Knowledge Technology (RSKT), 2006, pp. 685-692.

[7] Y. Tsai, C. Jiang, and Y. Huang, "A multi-scale crack fundamental element model for real-world pavement crack classification," Journal of Computing in Civil Engineering, vol. Accepted for publication, 2012.

[8] J. Laurent, D. Lefebvre, and E. Samson, "Development of a New 3D Transverse Laser Profiling System for the Automatic Measurement of Road Cracks," in the 6th Symposium on Pavement Surface Characteristics, Potoroz, 2008. 